\title{
"How Can We Talk about Patient-centered Care without Patients at the Table?" Lessons Learned from Patient Advisory Councils
}

\author{
Anjana E. Sharma, MD, Rachel Willard-Grace, MPH, Andrew Willis, BA, \\ Olivia Zieve, MSW, MPH, Kate Dubé, BA, Charla Parker, MPA, \\ and Michael B. Potter, MD
}

Context and Objective: Patient advisory councils (PACs) are a strategy for primary care clinics to engage patients in practice improvement. However, there is scant research on how PACs function. This study aimed to understand how PACs are organized and identify common challenges and perceived benefits of high-functioning PACs.

Setting and Population: Key informants identified 8 primary care clinics in California with highfunctioning PACs. Leaders from each of the 8 clinics nominated 1 clinic staff member and 1 PAC patient member to be interviewed.

Study Design: Semistructured, one-on-one interviews were conducted at each clinic site or by phone. Interviews were dual-coded using modified grounded theory. Common themes were identified that would be pertinent to the development of future best practices for running PACs.

Results: Common characteristics of high-functioning PACs included careful attention to participant recruitment, facilitation strategies guiding diverse personalities toward a common purpose, and assigning accountability for practice improvement projects. Interviewees identified a variety of positive outcomes that ranged from tangible improvements to the waiting area to a more patient-centered staff culture.

Conclusions: PACs show potential for promoting patient-centered practice improvements in primary care. Lessons learned from high-functioning PACs can inform a common set of strategies to assist practices in creating and sustaining effective advisory councils.(J Am Board Fam Med 2016;29:775-784.)

Keywords: Advisory Committees, Consumer Involvement, Patient Engagement, Quality Improvement, Patient-Centered Care, Medical Home

Patient engagement, a concept growing in popularity, can be defined as partnership between patients, families, and caregivers to improve health care. ${ }^{1}$ Typically, patient engagement is conceptu-

This article was externally peer reviewed.

Submitted 11 December 2015; revised 13 July 2016; accepted 18 July 2016.

From the Department of Family \& Community Medicine, University of California, San Francisco, School of Medicine (AES, RW-G, AW, OZ, KD, MBP); California Primary Care Association, Sacramento, CA (CP).

Funding: This project was supported by the National Center for Advancing Translational Sciences, National Institutes of Health (NIH), through UCSF-CTSI Grant Number UL1 TR000004. Its contents are solely the responsibility of the authors and do not necessarily represent the official views of the NIH. AES was supported by National Research Service Award T32HP19025.

Conflict of interest: AES has received travel reimbursement for dissemination of research findings at the Western Cli- alized as involving patients in their own individual care, but it can also be fostered at the level of the clinic or health care organization. ${ }^{2}$ For example, patients can be engaged in clinic-level improvement using approaches such as Patient Advisory Councils (PACs), which is a criterion of the $\mathrm{Na}$ tional Committee for Quality Assurance (NCQA) definition of the Patient-Centered Medical Home

nicians Network (WCN) Region IX Annual Meeting in June 2015, and served as a consultant for WCN to develop a toolkit based on research findings. The Center for Excellence in Primary Care received payment for a training and keynote address about patient engagement delivered by $\mathrm{Ra}-$ chel Willard-Grace in 2014. AW, OZ, KD, CP, and MBP have no conflicts to disclose.

Corresponding author: Anjana E. Sharma, MD, 1001 Potrero Avenue, Building 83, San Francisco, CA 94110. (E-mail: Anjana.sharma@ucsf.edu.) 
(PCMH). ${ }^{3}$ PACs are groups of patients, family members, and caregivers who meet on a regular basis to help identify practice improvement priorities and support practice improvement projects in collaboration with clinic staff members and leaders. However, only a third of certified PCMHs engage patients at the practice level through programs such as PACs. ${ }^{4}$ This may be because of the perceived effort required to organize PACs and limited data on the beneficial outcomes of such patient engagement activities. There is case-based evidence that inpatient advisory councils have had a positive effect on patient safety ${ }^{5}$ and readmissions ${ }^{6}$; however, less research has been performed in outpatient settings. One systematic review comprised primarily of case studies found that patient advisors helped improve clinic access and informational materials for patients. ${ }^{7}$ Another study found that when patient advisors worked with staff to set clinic priorities, those priorities were more aligned with the tenets of the PCMH than when staff set priorities alone. ${ }^{8}$ However, there is very little data on how PACs function within primary care clinics.

This study aimed to use qualitative methods to learn more about both patient and staff experiences with PACs in the primary care setting, and to expand our limited understanding of how PACs are established and maintained at primary care clinics.

\section{Methods \\ Setting}

This qualitative study was part of a mixed-methods needs assessment of patient engagement at the practice level led by the Center for Excellence in Primary Care (CEPC) and the San Francisco Bay Area Collaborative Research Network (SFBayCRN) at the University of California San Francisco, in partnership with 2 principal regional stakeholders in patient engagement: the Western Clinician's Network, a membership-based organization for community health center leaders in Arizona, California, Hawaii, Nevada, and the Pacific Islands, and the Center for Care Innovations, a nonprofit focused on facilitating innovation and quality improvement in safety net settings.

\section{Sample}

Through meetings with local and regional leaders in the field of patient engagement in practice transformation, the research team identified high-func- tioning PACs within Northern California. The study team used a positive deviance sampling strategy, based on the approach that surveying organizations that excel in a challenging arena can help identify best practices. ${ }^{9}$ With this approach, we met with key informants, primarily members of health care nonprofits with experience funding and facilitating patient engagement initiatives, who identified clinics with advisory councils that were considered "high-functioning." High-functioning PACs were considered to be so if they had PACs that had been operating for at least 6 months, were meeting on a regular basis, and had helped produce concrete improvements to clinic operations as identified by our key informants.

Clinics agreeing to participate nominated 1 clinic staff member and 1 PAC patient member to be interviewed. Staff members interviewed were those considered to be most involved with their PAC. All participants provided informed consent. The study protocol was reviewed by the University of California San Francisco Committee on Human Research and determined to be exempt.

\section{Data Collection}

An interview guide was designed by the study authors to explore how PACs promote patient engagement within clinics. The interview guide explored how PACs were formed and managed, and how clinics took advantage of PACs to improve clinic operations (See Appendix 1 for instrument). The interview guide was pilot tested and refined with input from project partners experienced in working with PACs.

Semistructured interviews were conducted individually in person or via telephone by a trained research assistant from May through August 2014. Interviews were conducted in English or Spanish. Interviewees were reimbursed with a $\$ 25.00$ gift card for their time. Sites were recruited and interviews were conducted until the research team observed that no new concepts were arising from interviews, suggesting that thematic saturation was reached. ${ }^{10}$

\section{Data Management and Analysis}

Interviews were transcribed, translated, and deidentified. Three members of the research group closely read all interviews and identified 16 main areas of interest that became the initial codes. Interviews were dual coded, in which each transcript was independently coded by 2 reviewers who dis- 
Table 1. Demographic Characteristics of Patient Advisory Councils (PACs)

\begin{tabular}{|c|c|c|c|c|c|}
\hline $\begin{array}{l}\text { Site } \\
\text { No. }\end{array}$ & Clinic Type & Duration of PAC & $\begin{array}{l}\text { Patient Members in } \\
\text { PAC, } \mathrm{n}\end{array}$ & $\begin{array}{l}\text { Meeting } \\
\text { Facilitator }\end{array}$ & $\begin{array}{l}\text { Meeting } \\
\text { Language }\end{array}$ \\
\hline 1 & $\begin{array}{l}\text { Public health internal } \\
\text { medicine health center, } \\
\text { FQHC }\end{array}$ & 1.5 years & 7 to 9 & Staff member & English \\
\hline 2 & $\begin{array}{l}\text { Public health family medicine } \\
\text { health center, FQHC }\end{array}$ & 4 years & 7 & Staff member & English \\
\hline 3 & FQHC & 10 months & 15 & Staff member & Spanish \\
\hline 4 & $\begin{array}{l}\text { Non-profit network of family } \\
\text { medicine FQHCs }\end{array}$ & 3 years & $\begin{array}{l}22 \text { patients representing } \\
\text { network of } 11 \text { practices }\end{array}$ & Staff member & English \\
\hline 5 & $\begin{array}{l}\text { Academic family medicine } \\
\text { health center }\end{array}$ & 5 years & 12 & $\begin{array}{l}\text { Staff and patient } \\
\text { co-chair }\end{array}$ & English \\
\hline 6 & $\begin{array}{l}\text { Public health community } \\
\text { health center, FQHC }\end{array}$ & 8 months & 12 & Staff member & Spanish \\
\hline 7 & Urban FQHC & 2 years & 15 & Staff member & Spanish \\
\hline 8 & Rural FQHC & 6 months & 12 & Staff member & English \\
\hline
\end{tabular}

FQHC, federally qualified health center.

cussed any discrepancies and revised the codebook to reflect their consensus. Data were analyzed using modified grounded theory, ${ }^{11,12}$ in which ideas were allowed to emerge, although we had some framework of concepts approaching the data based on our prior experience with PACs. The coding progressed in an iterative process, with the team meeting frequently to refine the codebook. Disagreements or discrepancies in coding were resolved through discussion between coders and additional discussion with other research group members until agreement was reached. Dual-coded interviews were entered into Atlas.ti (Version 7.0, Atlas.ti Scientific Software Development, GmbH). ${ }^{13}$

After dual coding all interviews, key findings were reviewed by the entire research group to confirm validation. Several major themes were derived from key findings. These themes were reviewed to identify variations between staff and patient respondents. The research group selected final themes based on prevalence among the interviewees and relevance for best practices.

\section{Results}

Of 13 nominated and invited clinics, 8 participated in the study, providing a total of 15 interviews. Two interviews were conducted at each site, 1 with a PAC staff member and 1 with a PAC patient member, with the exception of 1 clinic in which the clinic staff did not identify a patient leader to be interviewed. Interviews ranged from 45 to $60 \mathrm{~min}$ utes in length. Two patient interviews were con- ducted in Spanish. Four respondents (1 staff and 3 patients) self identified as Latino/a and 10 (7 staff and 3 patients) were female. PAC sized ranged from 7 to 15 members, and the duration of the PACs ranged from 6 months to 5 years. Of the 8 participating clinics, 5 served urban and 3 served rural areas. Seven of the organizations were family medicine specialty sites, and 1 was a general internal medicine site. Six of the sites were federally qualified health centers. Seven of the sites were safety-net clinics, and 1 site was a university-affiliated health center. A summary of the demographic details of clinic sites and PACs is listed in Table 1.

Four salient themes emerged from the interviews, roughly corresponding to the way a PAC is initially established (Logistics/Setup and Recruitment), the process of running a PAC meeting (Meeting Process), how PAC projects are implemented (Implementation/Followup), and the effect a PAC has on a clinic. A synthesis of proposed best practices culled from the data are listed in Table 2, with 4 prominent themes discussed in detail below. The interviewees also described an array of impactful projects done in conjunction with PACs, which are listed in Table 3 and exemplify how these PACs were high functioning.

\section{Theme No. 1: Recruitment Strategies Aim to Identify Patient Members with Strong Communication Skills Who Are Reflective of the Demographics of the Patients Served}

Recruitment of patient members for PACs typically involved publicizing the group in the clinic, obtain- 
Table 2. Proposed Best Practices for Patient Advisory Councils (PACs)

\begin{tabular}{l}
\hline Activity \\
\hline Logistics/Setup \\
$\begin{array}{c}\text { Membership of } 7-15 \text { people with te } \\
\text { limits, monthly meetings }\end{array}$ \\
Reimbursement/ incentives \\
Dedicated staff with protected time/ \\
resources for planning
\end{tabular}

Recruitment

Meeting Process

Implementation/Follow-up

ing staff and provider nominations, and conducting interviews of nominated or interested patients as a "vetting" process before inviting them to be a PAC served

Term limits; ongoing/rolling recruitment

Strong, trained facilitators

Staff members come to present to hear patient needs staff and patients contributing. right scale and rest of clinic
Engaged leadership

Supporting Quotes

"At first we were only three or four and now we're 12 or 13 members, so I think that if we had more members from the very beginning the changes could have been achieved more quickly."

"So for each, in compensation for like their time, each patient gets a gift card, a \$10 gift card, each meeting they come."

"There has to be sort of staff resource in place as far as is there someone who has the capacity to sort of lead this project. . I'm a full time staff person and I spend a lot of my work life helping to manage this... so it does take some time and effort."

"The top senior management team needs to be completely, $100 \%$ involved in the process and then get everyone excited and buy-in and help them through statistics and examples and patient stories."

So I think it was so important for us to sit down, to go through an interview process. Can you communicate with each other in a productive way?... And making sure that we had at least people with different experiences to bring, and then sharing with them really what our mission is about."

Focus on establishing a PAC that is reflective of the patient population

Support/training for new members

Patients bring their own experiences for practice improvement projects advisory council for "focus groups"

PACs reach out to broader clinic to

Clear definitions of roles for operationalizing projects, with both

Selection of the "right" project on the

Clear channels of communication and follow-up between advisory councils

"We do, you know, in the kind of instructions we give them to recruit patients or support we give them to recruit patients, we really ask them to make sure that they recruit someone that's sort of reflective of some of the demographics of their practice.

"We empower them through the Advisory Council... we really teach our advisors to do that so if they don't come with those skills, those are teachable skills."

"We do have term limitations on our council members. . 'cause we need fresh voices."

"You can create this trust where people like they don't feel like judged if like they bring an idea that's not good. . I would really work on having this like safety space where everybody feels they can share ideas and they won't be criticized."

"Patients also initiate projects. It kind of comes up more like I have this concern. And then we break down what might have led to that experience."

"Each meeting, there is always somebody from some-like the library or like today we had the lady with the other group. They come in and let us know what they're up to and what's going on with them."

"I think that in order that the council works things should be done together with the staff and I also suggested that all the staff members should be notified of the changes."

"Most of the time (clinic leadership) are very receptive to the ideas. And if for some reason they can't do anything about it right now, then they say, 'That's a great idea but right now we're holding off on it.' So then I reported that back to the Advisory Board."

"It's really important that the opinion goes somewhere so we try to be cognizant of trying to tell the council, "the last time you gave us this feedback. This is what member. Staff interviewees described wanting to recruit the "right patient" to participate in an advisory council: someone who could communicate happened to it and we think this is going to be the next steps we're going to take to making this change." 
Table 3. Examples of Practice Improvement Projects Established by Patient Advisory Councils

\begin{tabular}{|c|c|}
\hline Area of Impact & Examples \\
\hline Clinic physical space & $\begin{array}{l}\text { Improved waiting room chairs } \\
\text { Creation of wheelchair access } \\
\text { Clinic artwork } \\
\text { Posting of facesheets/bios of providers } \\
\text { Healthy vending machine food } \\
\text { Improved clinic signage }\end{array}$ \\
\hline Patient care/care communication & $\begin{array}{l}\text { Distribution of BP cuffs and scales for patients } \\
\text { Creation of patient feedback comment box } \\
\text { Patient emergency medical sheet } \\
\text { Improved visit summary } \\
\text { Improved Spanish-language materials } \\
\text { Patient-designed clinic brochure and welcome letter } \\
\text { Redesigned advance directive packet } \\
\text { Feedback on provider practice regarding giving bad news } \\
\text { Feedback on barriers to immunization } \\
\text { Creation of patient visit preparation tool } \\
\text { Patient calendar }\end{array}$ \\
\hline Clinic workflow/system & $\begin{array}{l}\text { Feedback on online patient portal/electronic medical record } \\
\text { Feedback on staff customer service } \\
\text { Designing PDSA (Plan-Do-Study-Act cycles) } \\
\text { Program to reduce patient no-shows } \\
\text { Improved telephone access and Spanish-language phone access } \\
\text { Interventions to reduce patient wait time }\end{array}$ \\
\hline Impact on clinic culture & $\begin{array}{l}\text { Staff are more receptive to patient feedback } \\
\text { Staff seeks patient feedback before initiating projects } \\
\text { Staff draw connections between improved patient experience of care and improved } \\
\text { patient adherence } \\
\text { Staff exposure to patient narratives } \\
\text { Changes in staff language more focused on patient experience } \\
\text { Initial staff resistance has abated } \\
\text { Movement to include patient members in other clinic programs and working groups } \\
\text { Improved sense of gratification and mission in work }\end{array}$ \\
\hline
\end{tabular}

BP, blood pressure.

ideas but also listen to others and who could work in a group even when there were differing opinions; "So in terms of patients, I guess picking or choosing patients to participate on an Advisory Board is a little tricky because you want someone who is able to share ideas but also listen to ideas..." Another staff member described multiple aims for their recruitment strategy:

"We learned... that you cannot just take anybody who applies, okay? Even though you want to. The thing that is most important is not what they think so much as how they express it. And so when you are vetting a patient or family member for a council position, it is really critical that they are not combative, that they are able to represent a number of voices and to listen to a number of voices and that they can be representative of people who are unlike themselves. And of course they all represent themselves and so hopefully you can try to get as diverse a council as you can, but that is also another challenge."

Although clinic staff members described wanting to recruit patients with specific communication skills, all 8 respondents also mentioned the goal to recruit members who would be reflective of the diversity of a clinic's patient population. Five staff and 1 patient mentioned the challenges of recruiting PAC members who were younger in age, members of ethnic minorities, working patients or parents, male members, those of lower literacy, those with chronic illness, and members of sexual minority groups. Establishing an advisory council that is reflective of the patient population served seemed 
to be a secondary goal for staff: "I think once we're established and solid and work out all the processes I am going to focus on what groups are we not representing." Challenges to improving diversity on councils included timing of meetings conflicting with work hours, lack of resources for interpreters, challenges for clinics to accommodate issues of physical frailty and disabilities affecting communication, and difficulty getting participation from younger patients and working families. Some patients described natural limitations of the perspective available within an advisory council and looked to strategies to collect broader patient feedback through comment boxes and surveys.

\section{Theme No. 2: Skilled Facilitation Is Instrumental for Successful and Productive PAC Meetings and Projects}

All 8 sites designated a staff member or staff liaison as meeting facilitator; at 1 site a patient co-chair functioned as cofacilitator along with a designated staff member. Facilitators were responsible for providing a safe space for all members to be involved, including reigning in members who had more dominant communication styles that could overpower meetings. The role of a staff person to facilitate smoothly was described by 1 patient as akin to an orchestra conductor:

"...If we're to be the model of patient advisory, we have to work out these kinks and to function. Like, I see the facilitator as a conductor and we are the musicians. One plays cello, one plays violin, one plays the piano. We all play our own music, everybody does, and the conductor is to synchronize and harmonize."

Skilled facilitators were described to effectively nurture project ideas from the PAC, but also seek patient feedback on projects and programs introduced by the clinic. Facilitators helped to translate patient experiences into realistic projects, and triaged possible project ideas and identified those that were not feasible: "It just seems that the staff members who are on the council and the clinicians who are on the council are helpful in guiding the patients to take their experience and putting it into some kind of practical project that both will find beneficial and useful." Facilitators also transmitted information about PAC meetings to the rest of the clinic.

Interestingly, many PAC facilitators described learning facilitation skills on the job without prior training. Over time, they developed confidence in their ability to mediate dominant members and usher group discussion more toward specific, achievable projects.

\section{Theme No. 3: Clarity between PAC Members and Clinic Staff Regarding Accountability Is Key for Project Implementation}

Respondents commonly discussed that PAC meetings foster conversation between patients and staff that generates ideas for practice improvement projects. However, there were differing expectations between staff and patients on who has primary responsibility for implementing clinic-level practice improvement projects. A majority of patients (6 patients, 1 staff) believed that it was the role of the clinic or staff liaison to work with the rest of the clinic to operationalize new projects. It was unclear whether this was an expectation that was promoted by clinic staff, but many patients described their staff or clinician liaison as taking responsibility for making progress on PAC projects: "[the clinician] is a really in-the-know person here in the clinic. I do not know exactly what her position is, but she's got enough pull that she gets things done. And she takes our suggestions and gets them done." Staff members were acknowledged as having more access to clinic logistics: "there's a project that is happening here, but it takes the staff to integrate it... So a lot of the decision or action plan falls on their shoulders."

In contrast, 6 respondents ( 4 staff, 2 patients) opined that patients should assume a share of the responsibility for moving projects forward. One staff member described how project selection and facilitation can foster more accountability when the patients are engaged: "So see, when they are passionate about it and when we can ask in a specific way to encourage them to be more involved, then it works. If we're not able to do that and if they are just not interested, then it will not work." Staff liaisons alluded to being overburdened with PACspecific work, and the possibility of increased patient support was one possible strategy to offset their workload. One patient commented on a middle ground of shared accountability between patients and staff: "If all the work had to be done by the clinic alone it would not be possible, so the patient has to cooperate too to make some changes, we have to work together, both patients and doctors.” 
Despite these challenges, the majority of respondents described a diverse array of successful clinic improvement projects implemented by PACs, including patient communication tools, clinical care, the clinic's physical space, and clinical workflows or systems (Table 3).

\section{Theme No. 4: By Spreading Awareness of a PAC and its Projects to the Clinic at Large, Clinics can Promote a Culture of Support and Buy-in for Patient Engagement Activities}

Of the many domains where patients and staff reported a PAC impact, a common finding was that over time, there was a notable change in "clinic culture" in which clinic staff became more receptive to patient-centered care, as well as more receptive to working with the PAC:

"From what I've experienced in the 4 years that I've been here now... [the PAC] makes a huge difference. It makes a huge difference about how providers think about how they interact with the patients, how the staff think about the work that they are doing, how patients feel involved in the care that they are receiving, versus being just something or a place they come to."

This change could happen early on, as a PAC begins to enact projects, or over time, as staff become more exposed to patient advisors and hear their experiences of care: "And then the culture has also been transitioning and some of the verbiage that I hear around in different meetings, managers are starting to say 'this came from the PACs'. So then nobody questions it as much, so they are gaining a positive reputation."

Through this clinic culture change, PACs were described as having more authority or "pull" at a clinic, such that they became a resource or sounding board for new ideas.

At sites that have invested in PACs, ongoing patient input was seen by staff as an essential component of achieving a culture of patient engagement: "How can we talk about patient-centered care without having patients at the table?"

\section{Discussion}

Key informants from these high-performing PACs identified a number of best practices for establishing and maintaining a PAC, derived from common successes and challenges from PAC-clinic partnerships. As one of the few existing studies of PACs, our findings provide guidance for health care organizations interested in establishing an advisory council.

Our findings provide insight that may help practitioners anticipate and address some of the challenges of PACs. Identifying patients with excellent communication skills as well as recruiting membership reflective of the patient population served emerged as dual and possibly competing priorities. By specifically recruiting excellent communicators, patients who may be more comfortable in facilitated meeting settings may be more likely to be nominated. These findings suggest that more time and resources are needed to recruit and support patients from hard-to-reach groups served by the clinic. Clinics may need to provide orientation and training to help prepare members who may be less experienced with group or meeting structures and processes.

Clinic staff also need support to provide the robust facilitation needed for successful PACs. Staff members described a lack of preparation on how to facilitate meetings and a sense of learning facilitation skills on the job. This stands in contrast with other patient group programs such as Centering Pregnancy or group visits for chronic pain, which provide formal facilitation training to providers or staff. ${ }^{14,15}$ As PACs become more common in the primary care setting, a future best practice could include trainings for staff liaisons in group facilitation and conflict resolution to best prepare for mediating diverse and dominant communication styles in meetings. Of note, all participating clinics had a staff member serve as either the group facilitator or co-facilitator. Future PACs may encourage patient members or other personnel to take the lead in PAC meeting discussions.

Our third finding identified dissonance between staff and patient perceptions of who is accountable to complete projects. Although staff members have a far better understanding of the inner logistics of a clinic and how to enact change, both staff and patient contributions are needed to operationalize projects. Having clear expectations regarding the time commitment and role of both the staff champion as well as patient members is a best practice that can help address this ambiguity.

It was an unexpected finding that so many staff members described a transformation in clinic-wide culture after working with a PAC. Patients did not speak to this effect, which may be due to their lack 
of exposure to a clinic's inner workings and staff meetings where such changes in dynamics may be most noticeable. This resonates with a recent study of Accountable Care Organizations (ACO) that found a positive association between ACO use of patient engagement activities and ACO leaders' belief that patient engagement has a positive effect. ${ }^{16}$

Although impactful projects were described by all respondents, the data did not reveal practical strategies to quantitatively evaluate the success of PACs, although some staff job responsibilities included monitoring patient experience measures. As PACs work with practice-improvement teams, developing metrics to assess PAC success is an important future area of inquiry. Although respondents did not describe metrics of success, there was overarching concordance that working with a PAC was worth the time it takes, which resonates with a recent cross-sectional survey conducted with practice leaders working in patient engagement at community health centers. ${ }^{17}$

Limitations of this analysis include a small sample size and focus on Northern California, although we did capture both urban and rural sites. Our results are most generalizable to primary care health centers primarily serving the safety net in which system leadership supports patient engagement efforts. Given that there is no validated metric to identify if the clinics that were nominated actually had "high-functioning" advisory councils or not, our sampling strategy was based on key informant opinion and may not have included all eligible sites. Because we interviewed 1 staff and 1 patient member from clinics and had 1 study site that did not nominate a patient interviewee, we may have encountered reporting bias by focusing on engagement champions and patient leaders within advisory councils who were likely predisposed toward speaking more positively about councils. Similarly, the sites we interviewed may not experience the same barriers and challenges faced by clinics with newer or less successful PACs, which means we may not have identified all salient barriers. However, our goal was to conduct a positive-deviance study focusing on "bright spots" within advisory council work and highlight successes in the field.

Our findings validate some of the case-based recommendations found in online resources, toolkits, and case studies about advisory councils. ${ }^{18}$ For example, multiple toolkits recommend a PAC membership size range that includes 7 to $15 \mathrm{mem}$ bers, meeting on either a monthly or quarterly basis, and offering reimbursements. ${ }^{19,20}$ As discussed by our respondents, recruitment based on communication skills as well as to ensure a council reflects the patient population served are highlighted in online resources. ${ }^{21}$ The positive culture change catalyzed by PACs is also mentioned as a benefit at other health care organizations who have invested in patient engagement. ${ }^{20}$

\section{Conclusions}

This formative study found a number of practices that may be essential for successful PACs in primary care settings. These practices include an intentional recruitment strategy, providing facilitator training and support, making sure clinic and PAC have clear workflows establishing accountability for project implementation, and promoting awareness of PAC contributions. In our study, both patients and staff working with high-functioning PACs believed that their activities had a positive influence on clinic programs, clinic culture, and patient care. As the field of clinic-level patient engagement evolves, these practices can be tested in future interventions to understand more about the demonstrable effect of PACs.

Acknowledgements: We thank the patient partners and clinic staff who participated in interviews. We also thank Susannah Brower at Center for Care Innovations for her help in identifying participants and providing vision for this study; and finally, we thank Dr. David Thom for his thoughtful review of the manuscript and findings.

\section{References}

1. Rickert J. Patient-centered care: What it means and how to get there. Health Affairs Blog. Available at: http://healthaffairs.org/blog/2012/01/24/patientcentered-care-what-it-means-and-how-to-getthere/.

2. Carman KL, Dardess P, Maurer M, et al. Patient and family engagement: a framework for understanding the elements and developing interventions and policies. Health Aff (Millwood) 2013;32:223-31.

3. NCQA. PCMH 2011-PCMH 2014 Crosswalk. 2015.

4. Han E, Hudson Scholle S, Morton S, Bechtel C, Kessler R. Survey shows that fewer than a third of patient-centered medical home practices engage patients in quality improvement. Health Aff (Millwood) $2013 ; 32: 368-75$. 
5. Longtin Y, Sax H, Leape LL, Sheridan SE, Donaldson L, Pittet D. Patient participation: Current knowledge and applicability to patient safety. Mayo Clinic Proc 2010;85(1):53-62.

6. Initiative leads to $11 \%$ drop in $\mathrm{HF}$ readmissions. Hosp Case Manag 2013;21(4):48-9.

7. Crawford MJ, Rutter D, Manley C, et al. Systematic review of involving patients in the planning and development of health care. BMJ 2002;325(7375):1263.

8. Boivin A, Lehoux P, Lacombe R, Burgers J, Grol R. Involving patients in setting priorities for healthcare improvement: a cluster randomized trial. Implement Sci 2014;9:24.

9. Bradley EH, Curry LA, Ramanadhan S, Rowe L, Nembhard IM, Krumholz HM. Research in action: Using positive deviance to improve quality of health care. Implement Sci 2009;4:1-11.

10. Marshall MN. Sampling for qualitative research. Fam Pract 1996(6);13:522-5.

11. Scott K, Dana H. Clarifying analysis and interpretation in grounded theory: Using a conditional relationship guide and reflective coding matrix. Int $\mathrm{J}$ Qual Methods 2008;7:1-15.

12. Walker D, Myrick F. Grounded theory: An exploration of process and procedure. Qual Health Res 2006;16(4):547-59.

13. Atlas.ti [computer program]. Version 7. Berlin, 2015.

14. Walker DS, Worrell R. Promoting healthy pregnancies through perinatal groups: A comparison of CenteringPregnancy ${ }^{\circledR}$ group prenatal care and childbirth education classes. J Perinat Educ 2008; 17(1):27-34.

15. Eisenstat Sea. Putting group visits into practice: A practical overview to preparation, implementation, and maintenance of group visits at Massachusetts General Hospital. 2012. Available from: http://www.massgeneral.org/stoecklecenter/assets/pdf/group_visit_ guide.pdf.

16. Shortell SM, Sehgal NJ, Bibi S, et al. An early assessment of accountable care organizations' efforts to engage patients and their families. Med Care Res Rev 2015;72(5):580-604.

17. Willard-Grace R, Sharma AE, Charla Parker MP, Potter MB. Engaging patients as partners in practice improvement: A survey of community health centers. JCOM 2016;23(7).

18. Newton WP, Atkinson H, Parker DL, Gwynne M. Bringing patients into the patient-centered medical home: Lessons learned in a large primary care practice. N C Med J 2015;76:190-3.

19. Institute for patient and family-centered care. Tools to foster the collaboration with patient and family advisors. 2015. Available from: http://www.ipfcc.org/tools/ downloads-tools.html. Accessed December 4, 2015.

20. Aligning Forces for Quality. Engaging patients in improving ambulatory care: A Compendium of tools from Maine, Oregon, and Humboldt
County, California. March 2013. Accessed December 4, 2015.

21. National Institute for Children's Health Quality. Creating a Parent and Family Advisory Council (PFAC): Toolkit. 2015. Available from: http://www.nichq.org/ sitecore/content/medical-home/medical-home/ resources/pfac-toolkit. Accessed December 4, 2015.

\section{Appendix 1: Interview Guides}

Patient engagement as transformation partners in

\section{primary care practices}

Interview/focus group guide

Confirm permission to record once recorder is turned on

Before we begin, may I have your permission to record this interview? As a reminder, you are free to ask me to turn off the recorder at any time.

\section{Staff Version}

\section{Warm-up}

1. How are you involved in practice improvement at your health center?

2 . What is your connection with patient engagement?

3. How long have you been involved in this capacity? What motivated you to get involved?

\section{Body of interview}

4. Tell me about the first times that you worked with patients to improve something at your health center.

Potential probes: What kind of concerns did you feel about working together with patients in this way? What motivated you to work together? What surprises did you experience along the way? What was the outcome of your work together?

5. Has there been a turning point or has anything changed during your experience?

6. Tell me about a time when you tried to engage patients in a practice improvement effort, and it did not work well. What happened?

7. Tell me about the opposite type of experience: what was a time when patient engagement worked really well? What made that possible?

8. What skills do you think that staff and patients need to gain to partner effectively?

9. What are the biggest barriers at your clinic to meaningful patient engagement?

10. How has patient input changed your clinic?

Potential probes: Services? Policies? Attitude? 
11. To what degree do you think your board is representative of patients? What voices do you think are underrepresented?

12. Looking back, what would you do differently?

13. Something I've struggled with is whether cohesion of a group is due to the groups make-up, or how similar the views of patients are. What do you think about this?

\section{Cool-down}

12. What advice would you offer to other practices wanting to work with patients to improve the way their clinics work? What lessons have you learned?

13. If you could dream big, what would you like patient engagement to look like in 10 years in your clinic?

14. Can you recommend patient leaders on your board I can speak with?

15. Can you recommend anyone I can speak with at another clinic doing this type of work?

What would be useful for you to learn from this needs assessment? What would you like to know from other health clinics doing this work?

\section{Patient engagement as transformation partners in primary care practices \\ Interview/focus group guide \\ Confirm permission to record once recorder is turned on \\ Before we begin, may I have your permission to record this interview? As a reminder, you are free to ask me to turn off the recorder at any time.}

\section{Patient Version}

\section{Warm-up}

1. How are you involved in practice improvement at your health center?

2. What is your connection with the clinic?

3. How long have you been working with the clinic in this capacity?

\section{Body of interview}

4. Tell me about the first times that you worked with staff to improve something at your health center.

Potential probes: What kind of concerns did you feel about working together with staff in this way? What motivated you to work together? What surprises did you experience along the way? What was the outcome of your work together?

(what happens with suggestions from suggestion box?)

5. Has there been a turning point during your experience? Or, has there been a 'light bulb moment' that has caused a transition or changed your experience or commitment?

6. Tell me about a time when you tried to engage clinic staff in a practice improvement effort, and it did not work well. What happened?

7. Tell me about the opposite type of experience: what was a time when working together with clinic staff worked really well? What made that possible?

8. What skills do you think that staff and patients need to gain to partner effectively?

9. What are the biggest barriers at your clinic to meaningful patient engagement?

10. How do you think that your input has changed your clinic?

Potential probes: Services? Policies? Attitude?

11. Looking back, what would you do differently?

\section{Cool-down}

12. If someone in a primary care practice were to ask you what they could do to work with patients to improve the way their clinics works, what advice would you offer? What are your lessons learned?

13. If you could dream big, what would you like patient engagement to look like in 10 years in your clinic? 